\title{
Sleep and cardiovascular health needs further research in the general population
}

\author{
Xianchen Liu ${ }^{1}$
}

Published online: 13 May 2021

(c) Japanese Society of Sleep Research 2021

Cardiovascular diseases (CVDs) are the leading cause of death, accounting for $31 \%$ of all deaths globally. More than $80 \%$ of deaths from CVDs are due to heart attack and stroke. Multiple genetic, biological, behavioral, and psychosocial factors can increase risk of CVDs directly and/or indirectly. Enough and good sleep is essential for health and wellbeing. Growing evidence has shown that sleep problems including inadequate sleep duration (short or long sleep), poor sleep quality, sleep disturbances, sleep-related breathing disorders, and irregular sleep patterns are associated with increased risk of CVDs [1-5]. Sleep problems may have direct effects on CVDs or indirect effects via elevated risk of high blood pressure, high blood glucose, high blood lipids, and overweight/obesity [4, 6].

Although many epidemiological studies have been conducted, the findings are inconsistent. For example, the relationship between sleep duration and risk of CVDs has been demonstrated to be a u-shaped curve, i.e., both short and long sleep durations are associated with elevated risk of CVDs. However, a recent meta-analysis demonstrated that longer sleep may be more associated with adverse outcomes compared with shorter sleep [2]. Inconsistent findings are largely due to differences in study design (retrospective, cross-sectional, or prospective), target populations, sample sizes, measures used to assess sleep, definitions of sleep problems and cardiovascular outcomes, and confounding factors adjusted for in previous studies.

In the current issue of Sleep and Biological Rhythms, Dr. $\mathrm{Li}$ and coauthors conducted a systematic review and metaanalysis to demonstrate the longitudinal associations of sleep duration and sleep quality with risk of coronary heart disease (CHD) in the adult population [7]. The meta-analysis combined and analyzed 11 prospective epidemiological

Xianchen Liu

xclpsymd@gmail.com

1 Center for Public Health Initiatives, University of Pennsylvania, Philadelphia, PA 19104, USA studies with 769,452 adults and 14,769 patients with CHD events. In the classical meta-analysis, either short sleep duration ( $<7 \mathrm{~h} / \mathrm{night}$ ) or long sleep duration ( $>8 \mathrm{~h} / \mathrm{night}$ ) was associated with $13 \%$ increased risk of CHD compared with sleep 7-8 h. However, the Bayesian network meta-analysis showed that poor sleep quality was associated with $20-40 \%$ increased risk of CHD, independent of sleep duration. The authors concluded that poor sleep quality rather than inadequate sleep duration was associated with great morbidity and mortality of CHD.

The major strengths of the meta-analysis are: (1) both classical and Bayesian network meta-analyses were conducted; and (2) the effects of poor sleep quality were examined with sleep duration being stratified. In addition to the common limitations of many meta-analyses, the specific limitations of the analysis are: (1) only 11 studies were included in the analysis and only 4 studies evaluated both sleep quality and sleep duration; (2) sleep quality and sleep duration in the studies included were assessed based on self-reports or only 1-2 simple questions; and (3) other sleep variables and many non-sleep potential confounders were not controlled for in the studies included.

Despite these limitations, this meta-analysis provides additional evidence for the inadequate sleep duration and poor sleep quality-CHD link and emphasizes the role of poor sleep quality in increasing risk of CHD. Given the limitations of previous studies on the sleep-CVD link, further epidemiological studies are needed, including but not limited to:

1. Further large-scale longitudinal studies or registries instead of secondary data analyses are warranted to specifically investigate the association between sleep and CVDs.

2. Long-term follow-up with sleep assessments at multiple time points is needed because sleep duration and sleep disturbances may change over time. 
3. Multiple dimensions of sleep should be measured and included for statistical analyses to determine which sleep variables are independent predictors because sleep variables such as sleep duration, sleep disturbances, and sleep quality are highly correlated. The following sleep variables should be considered in future studies: weekday and weekend sleep duration, night and daytime sleep duration, napping, sleep patterns/sleep regularity, sleep quality, sleep efficiency, insomnia, sleep-related breathing disorders, daytime sleepiness, and other sleep problems.

4. Both subjective and objective sleep measures should be used as subjective and objective sleep measures are usually low-moderately correlated and they may reflect different dimensions of sleep and have different impacts on health. Validated questionnaires/scales should be used instead of only a few questions to assess subjective sleep. Advanced mobile sleep monitors such as smart phone for convenience to use are recommended to assess objective sleep.

5. What is adequate sleep duration-How short is short and how long is long? Sleep $<7 \mathrm{~h},<6 \mathrm{~h}$, or $<5 \mathrm{~h} /$ night has been used to define short sleepers while sleep $>8 \mathrm{~h}$ or $9 \mathrm{~h}$ has been used to define long sleepers in previous studies. Mixed findings may be due to different cutoffs used. On the other hand, sleep duration and sleep need may be different from person to person. Using the same cutoff for all individuals may not be appropriate. It is important to define adequate sleep duration based on individual sleep need/preference [5].

6. Mediators and moderators need to be identified to better understand the pathways between sleep and CVD risk for developing effective intervention programs. Potential mediators include cardiometabolic risk factors, unhealthy lifestyle behaviors, overweight/obesity, inflammation, endothelial dysfunction, chronic life stress, and anxiety/depression $[4,6]$.
7. Population-based intervention programs of sleep hygiene should be developed and clinically evaluated in the community setting to investigate whether and to what extent sleep intervention could reduce the risk of CVDs.

\section{References}

1. Huang T, Mariani S, Redline S. Sleep irregularity and risk of cardiovascular events: the multi-ethnic study of atherosclerosis. J Am Coll Cardiol. 2020;75(9):991-9.

2. Kwok CS, Kontopantelis E, Kuligowski G, et al. Self-reported sleep duration and quality and cardiovascular disease and mortality: a dose-response meta-analysis. J Am Heart Assoc. 2018;7(15):008552.

3. Zuraikat FM, St-Onge MP, Makarem N, Boege HL, Xi H, Aggarwal B. Evening chronotype is associated with poorer habitual diet in us women, with dietary energy density mediating a relation of chronotype with cardiovascular health. J Nutr. 2021. https://doi. org/10.1093/jn/nxaa442.

4. Daghlas I, Dashti HS, Lane J, et al. Sleep duration and myocardial infarction. J Am Coll Cardiol. 2019;74(10):1304-14.

5. Jike M, Itani O, Watanabe N, Buysse DJ, Kaneita Y. Long sleep duration and health outcomes: a systematic review, meta-analysis and meta-regression. Sleep Med Rev. 2018;39:25-36.

6. Liu X, Forbes EE, Ryan ND, Rofey D, Hannon TS, Dahl RE. Rapid eye movement sleep in relation to overweight in children and adolescents. Arch Gen Psychiatry. 2008;65(8):924-32.

7. Zeng R, Jiang Y-T, Chen T-W, Guo D-D, Li R. Longitudinal associations of sleep duration and sleep quality with coronary heart disease risk among adult population: classical meta-analysis and Bayesian network meta-analysis. Sleep Biol Rhythms. 2021. https://doi.org/10.1007/s41105-021-00312-1.

Publisher's Note Springer Nature remains neutral with regard to jurisdictional claims in published maps and institutional affiliations. 